\title{
Reprint
}

\section{A Dynamically Adaptable Polling Scheme for Voice Support in IEEE802.11 Networks}

E. Ziouva and T. Antonakopoulos

Computer Communications

Vol. 26 No. 2, FeBruary 2003, pp. 129-142

Copyright Notice: This material is presented to ensure timely dissemination of scholarly and technical work. Copyright and all rights therein are retained by authors or by other copyright holders. All persons copying this information are expected to adhere to the terms and constraints invoked by each author's copyright. In most cases, these works may not be reposted or mass reproduced without the explicit permission of the copyright holder. 


\title{
computer

\section{A dynamically adaptable polling scheme for voice support in IEEE802.11 networks}

\author{
Eustathia Ziouva, Theodore Antonakopoulos* \\ Department of Electrical Engineering and Computers Technology, University of Patras, 26500 Rio-Patras, Greece
}

Received 4 May 2001; revised 2 April 2002; accepted 1 May 2002

\begin{abstract}
This paper presents a new dynamically adaptable polling scheme for efficient support of voice communications over an IEEE802.11 network. The proposed Cyclic Shift and Station Removal polling scheme is implemented only on the Access Point of each Basic Service Set without requiring any modification on the existing access protocol. This polling scheme increases the number of conversations in case of silence detection, while maintains high voice quality. By considering constant bit rate digitized voice traffic, the paper presents a discretetime Markov chain model, that is, used to analyze the performance of the IEEE802.11 Point Coordination Function in terms of maximum number of supported conversations, when silence detection is used at the mobile terminals. The paper also determines how the parameters of the proposed polling scheme have to be related to the silence detector hangover.
\end{abstract}

(C) 2002 Elsevier Science B.V. All rights reserved.

Keywords: IEEE802.11; Wireless LAN; Centralized control; Speech communication

\section{Introduction}

The Point Coordination Function (PCF) is an optional access method within the IEEE802.11 MAC sublayer architecture [1] that provides contention free packets transmission. PCF is based on a polling procedure controlled by a Point Coordinator (PC) operating at the Access Point (AP) of each Basic Service Set (BSS). Such centralized control is more suitable to support time-bounded traffic than the Carrier Sense Multiple Access with Collision Avoidance (CSMA/CA) protocol employed by the Distributed Coordination Function (DCF) for supporting asynchronous traffic.

Wireless LAN technology is rapidly becoming a crucial component of networks connecting mobile users with multimedia capabilities. IEEE802.11 Task Group E works to enhance the current 802.11 MAC in order to support LAN applications with QoS requirements [2]. There are several papers that analyze the performance of IEEE802.11 LANs. Visser [3] simulated the combination of speech and data traffic over an IEEE802.11 network using statistical multiplexing, assuming that the voice activity occurs between stations belonging to different BSSs. It was concluded that

\footnotetext{
* Corresponding author. Tel.: + 30-61-997-346; fax: + 30-61-997-342.

E-mail address: antonako@ee.upatras.gr (T. Antonakopoulos).
}

the number of supported voice conversations is low and of poor performance. In Refs. [4,5], Crow's simulations suggest that an echo canceller is required for handling on/ off speech traffic exchanged among different BSSs. However, Crow sets a bound of $500 \mathrm{~ms}$ to the delay that the voice packets experience from the time the first bit is generated at the transmitting station, until the last bit is received at the $\mathrm{AP}$ and he does not consider the end-to-end delay. A similar approach is adopted in Ref. [6], which evaluates the IEEE802.11 PCF mode for supporting realtime traffic. In Ref. [7], the PCF mode is used to carry telephony traffic from 802.11 phones to wired, cellular or Internet phones, the maximum number of supported voice calls and the end-to-end delay are calculated. Other papers suggest the use of DCF for real-time traffic although the CSMA/CA protocol cannot guarantee delay bounds. Sobrino [8] proposed a distributed jamming scheme to transmit voice packets over an ad hoc IEEE802.11 network. Voice stations sort their access rights by jamming the channel with pulses of energy before sending their voice packets. Since this scheme does not conform to the IEEE802.11 standard, it is difficult to be used with existing implementations. Romans [9] presents a hybrid protocol for wireless LANs, which combines both TDMA access mechanism to support voice and CSMA/CA access mechanism to support data. This hybrid protocol is designed 


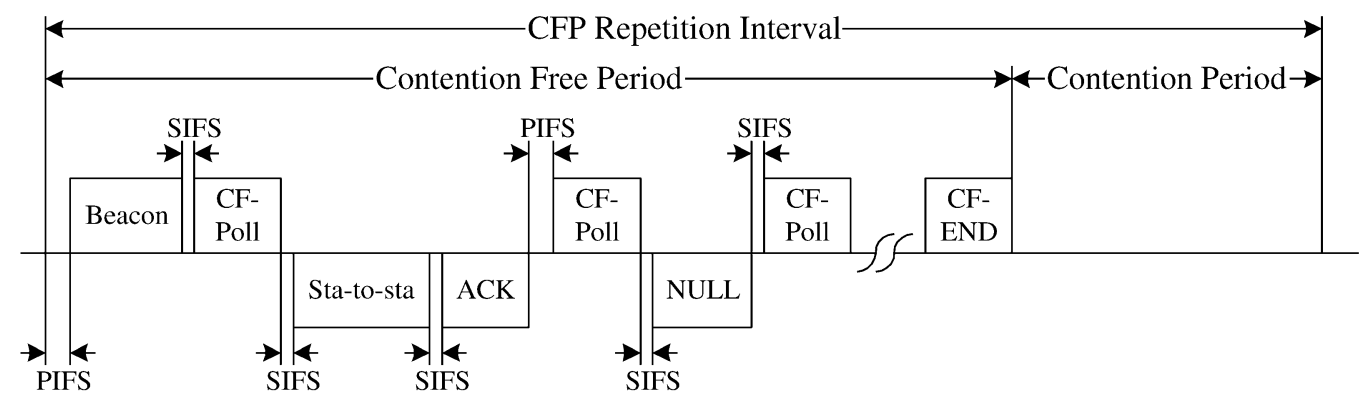

Fig. 1. Voice transmission over an IEEE802.11 BSS network.

for use on a frequency hopping system and offers only up to four reliable voice connections. In Ref. [10], a modified DCF access mechanism is proposed in order to provide realtime applications. Sheu [11] proposed another modified protocol to provide asynchronous and multimedia traffic over IEEE802.11 ad hoc wireless LANs. However, the proposed protocols in Refs. [10,11] are not designed for real-time traffic exchange between stations belonging to different BSSs, where all real-time packets have to be transferred through the AP of the BSS. Another approach, which adapts the Power-Saved (PS) mode of the IEEE802.11 specifications for carrying the voice traffic produced by G.729 speech coders between different BSSs, is presented in Ref. [12]. Moreover, papers [10-12] demand considerable modifications to the IEEE802.11 specifications that may potentially impact backward compatibility and future adoption of the IEEE802.11 wireless LAN. Finally, Zahedi [13] integrates voice and data with Transmission Control Protocol/Internet Protocol (TCP/IP) protocol operating in a CSMA environment and adopts the Improved Multiband Excitation (IMBE) low speed vocoder.

Both DCF and PCF have their strengths and weaknesses. DCF works better under low traffic conditions, but its efficiency drops considerably in densely populated BSSs. On the other hand, PCF has better performance under high offered load conditions, it is better suited for networks, where BSSs are carefully planned not to overlap and does not have a scaling problem. Although PCF introduces high overhead in each transaction, it can satisfy time-bounded requirements if it is properly adjusted. In this paper, we focus on the analysis of PCF and we propose a new dynamically adaptable scheme for efficient support of voice communications in IEEE802.11 BSSs. This dynamically adaptable scheme is implemented on APs and does not require any modifications on the mobile terminals access mechanism. We consider voice activities inside the BSS and channel bit rate of 5.5 and $11 \mathrm{Mbps}$ (the high data rate extension of the IEEE802.11 standard [14]). We estimate an upper bound on the number of voice conversations that a BSS can handle, while keeping low voice packet delay and guaranteeing predetermined minimum bandwidth for data traffic. Our studies were performed using $64 \mathrm{kbps}$ Pulse Code Modulation (PCM) voice coding and results are derived for scenarios with and without silence detection.
Section 2 describes the integration of voice and data in an IEEE802.11 BSS, the voice station model, that is, used in our system and the proposed polling scheme. In Section 3 , we present an analytical approach to derive the maximum number of voice stations in case of CBR voice traffic, while the bandwidth available to data stations is limited to a minimum value, and we continue our analysis using silence detection and the proposed polling scheme. Initially, we evaluate the performance of the proposed polling scheme for error-free transmissions and we proceed by examining the effects of transmission errors. Finally, Section 4 presents extensive numerical results, which demonstrate the improved performance of the proposed method.

\section{PCF operation and the dynamically adaptable polling scheme}

We consider a BSS network that employs PCF and DCF functions using a time-sharing mechanism. When DCF is used, the network is in a Contention Period (CP) and the stations must compete for gaining access. When PCF is used, the network is in a Contention Free Period (CFP) and the stations do not compete for transmitting their frames. Each CFP begins with a Beacon frame transmission and alternates with the $\mathrm{CP}$. The PC generates its Beacon frame at predetermined time instants, which are defined by the Contention Free Repetition Interval (CFPRI) parameter and determine the so-called Target Beacon Transmission Time (TBTT). The length of CFP is based on the available traffic and the size of the polling list. The PC may terminate any CFP round at or before a maximum duration, called CFPmaxDuration. The CFP is foreshortened, if at the nominal Beacon transmission time, the medium is busy due to DCF traffic. In this case, the PC ends the CFP no later than TBTT plus the value of CFPmaxDuration. Since the actual duration of CFP and CP may vary, the IEEE802.11 standard defines a minimum CP duration and a maximum value for the amount of time that the CFP is foreshortened, which is the maximum extension that the previous CP may experience due to busy medium conditions. All timing parameters that determine the coexistence of PCF and DCF are contained in the Beacon frame. 


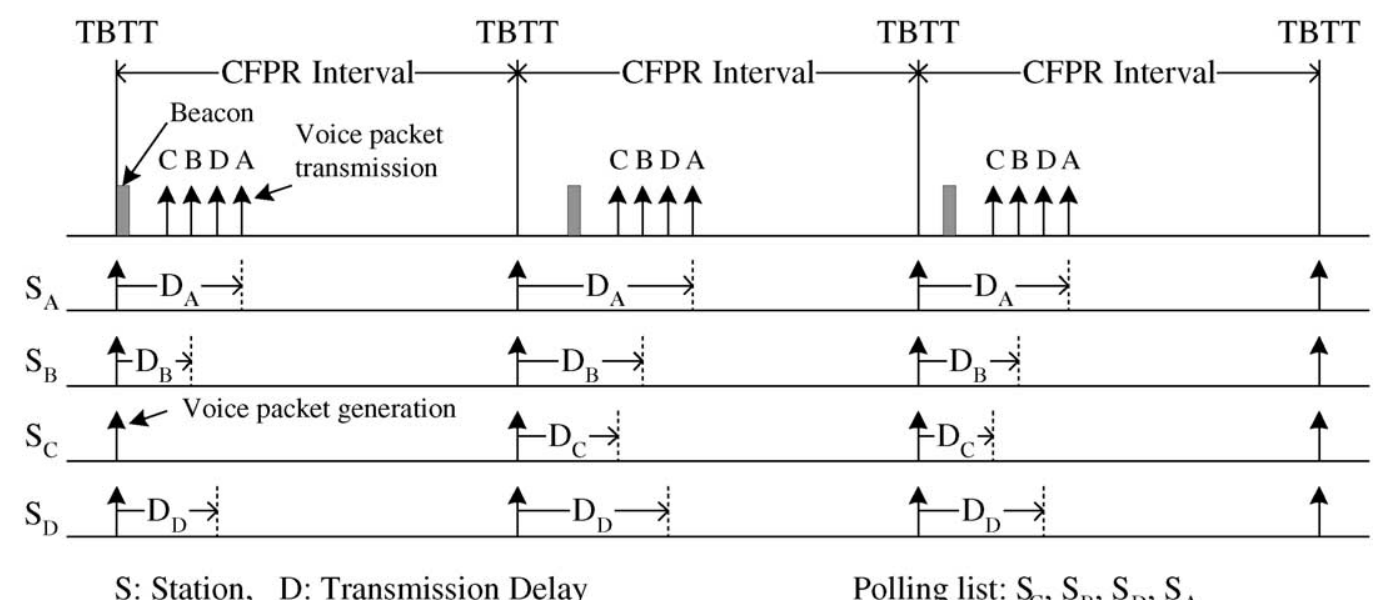

Fig. 2. Voice traffic management.

Each voice station desiring to make a voice call issues a request to the PC and if the request is accepted by the PC and the called station, the transmitting and receiving stations are placed on the PC's polling list. When the CFP starts, the PC sends a CF-Poll to the first station in the polling list. This station sends its voice packet to the other station in the BSS, no later than SIFS time after receiving the CF-Poll from the $\mathrm{PC}$. When the destination station receives the voice packet, a DCF ACK frame is returned to the source station and the PC waits a PIFS interval following the ACK frame, before polling the next station in the polling list. If the polled station has no frame to send, the response shall be a NULL frame. The PC terminates the CFP with a CF-END frame. Fig. 1 depicts the rules under which voice packets are transmitted during CFP.

In this work, we assume that the voice stations use PCM at $64 \mathrm{kbps}$ and a voice packet is generated every CFPR interval. A voice packet is transmitted each time the station is being polled by the PC. If a new packet is generated before the previous packet has been transmitted, the older packet is discarded. We consider that all stations on the polling list are polled no more than once per CFP. That procedure limits the probability of lost packets, since the time between two successive polling instants of the same station is close to the voice packet generation interval. This time is not exactly equal to the packet generation interval due to the fact that the CFP is sometimes reduced. By considering that a new voice packet is generated at every TBTT instant, as shown in Fig. 2, each voice packet suffers a variable delay, until the polling instant of its station arrives, but the packet is not lost. Furthermore, the delay a voice packet experiences cannot exceed the CFPR interval duration. The consideration that new voice packets are generated at the same instant simplifies the evaluation of the packet delay variations, since they are restricted by an upper bound-the CFPR interval. The synchronization of all stations in a BSS is achieved with the Timing Synchronization Function (TSF) defined in IEEE802.11 Standard. All stations maintain a TSF timer, that is, set to the timestamp value contained in the received Beacon frames sent by the $\mathrm{AP}$ and therefore every station can determine the correct TBTT instant. Since voice is delay sensitive, the CFPR interval is limited to values less than $25 \mathrm{~ms}$.

\subsection{Voice source model}

A voice source alternates between talk spurts and silent periods. In case of using silence detection, a station, that is, in a talk spurt, generates voice packets periodically, but if it is in a silent period, no voice packets are generated. By exploiting this characteristic, the PCF can handle more voice transmissions. We adopt the discrete-time version of a well-known model proposed in Ref. [15], according to which each voice source is modeled by a two-state discretetime Markov chain. The duration of a voice talk spurt fits the exponential distribution with average $d_{\mathrm{t}}$ equal to $400 \mathrm{~ms}$ and the duration of silent periods also follows the exponential distribution with average $d_{\mathrm{s}}$ equal to $600 \mathrm{~ms}$ [16]. The probability $p_{\mathrm{t}}$ that a voice station is in a talk spurt is given by $p_{\mathrm{t}}=d_{\mathrm{t}} /\left(d_{\mathrm{t}}+d_{\mathrm{s}}\right)[17]$.

Furthermore, the silence detectors use a technique to avoid sudden end-clipping of speech and to bridge short speech gaps, such as those due to stop consonants, the so-called hangover. Depending on the hangover, the mean spurt and silent length fall into two regions. If the hangover is around $200 \mathrm{~ms}$, mean spurt and silent length is in the order of $1-2 \mathrm{~s}$ $[5,15,17]$. If the hangover is small, mean spurt is from 200 to $400 \mathrm{~ms}$ and the mean silent length is from 500 to $700 \mathrm{~ms}$ [16, 18]. In this paper, we assume a small hangover (of the order of a few tens of milliseconds), since it is commonly used in many silence detectors. If at a TBTT instant a station is in a talk spurt, at the next PCF round the station generates a voice packet, that is, transmitted when the PC polls the station. If at a TBTT instant a station is in silent period, no voice packet is generated at the next PCF round and the station transmits a NULL frame, when it is polled. We consider that a station makes at most one state transition during a CFPR interval. 


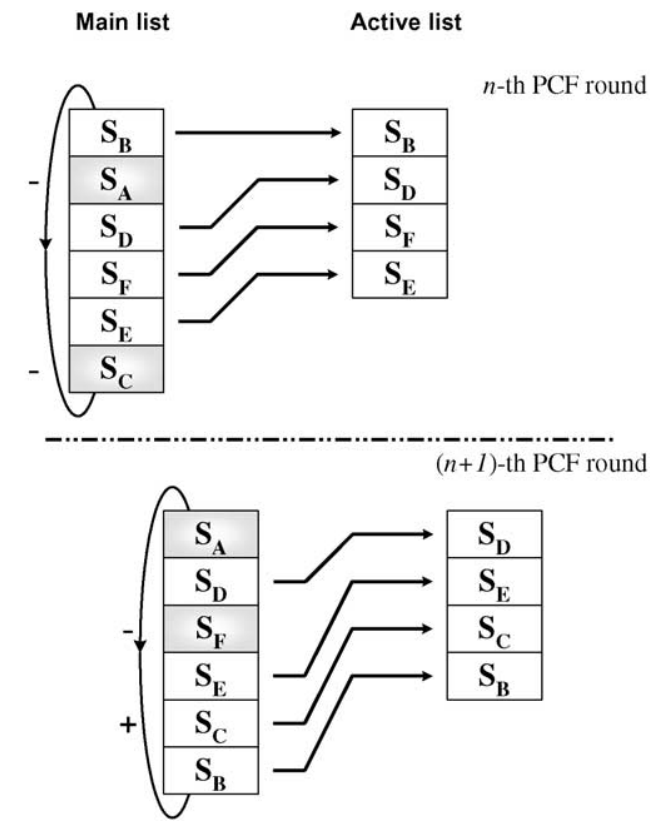

Fig. 3. Implementation of the CSSR polling scheme on an AP.

\subsection{The dynamically adaptable polling scheme}

The use of silence detection results to the increase of the number of voice stations supported by the network, compared to the case of not using silence detection. Whenever the PC cannot complete its polling list during a CFP period, we assume that the PC rejects the stations that cannot be polled during the current polling round and restarts the polling sequence with the first station on its polling list at the next CFP round. According to Refs. [16, 19], a bound of $1 \%$ packet loss can be tolerated without adversely affecting the subjective quality of the supported voice service and this bound determines the increase on the number of voice stations handled by PCF, when silence detection is employed. Obviously, the rejected stations of the polling list experience packet loss rates and the last station on the polling list experiences the highest packet loss rate. It seems that a solution to this problem may be given if at the next CFP round the PC continues the polling sequence from where it was stopped. In this case, the distance of two successive polling instants of the same station may be greater than the voice packet generation interval, and that causes higher packet loss rate to all stations in the polling list.

Restarting the polling list results to unfair stations support, since all rejections are not equally distributed to all stations. Therefore, in order to spread the rejection rate to all active stations in the network and by exploiting the characteristics of the voice model, we propose a new polling scheme, which is implemented only at the AP and does not require any modification on the access protocol used by the mobile terminals. The basic functionality of this dynamically adaptable polling scheme is the following.
- At the beginning of each CFP round, the PC cyclically shifts the stations on its polling list, so the first station at the previous round becomes the last station at the current round and all other stations advance one position towards the start of the polling list.

- At the beginning of each CFP round, the polling starts from the beginning of the new polling list.

- The PC stops polling a station temporarily (for a few PCF rounds), when the start of a silent period for that specific station is detected by the transmission of a NULL frame. Thus, the PC maintains two logical polling lists, a main polling list that contains all stations, irrespective of their state and an active polling list (a subset of the main polling list), which contains only the stations that have to be polled at the next PCF round.

During a PCF round, the PC polls sequentially the stations according to the current active polling list. In each round, the PC marks on its main polling list which stations enter silent state and which previously silent stations become active again. The PC also cyclically shifts its main list one position towards its start and then determines the active polling list for the next PCF round. An example of the Cyclic Shift and Station Removal (CSSR) polling scheme is shown in Fig. 3, where six stations compose the PC's main polling list. In the (n) PCF round, stations $\mathrm{S}_{\mathrm{A}}$ and $\mathrm{S}_{\mathrm{C}}$ are in silent state and do not participate in the active polling list. In the $(n+1)$ PCF round, all stations in the main list are cyclically shifted one position towards its start, station $S_{C}$ becomes active and station $S_{F}$ enters silent state. Therefore, the new active polling list is constructed by the new main polling list and $\mathrm{S}_{\mathrm{D}}$ becomes the first polled station.

A major contribution of the proposed polling scheme is the use of the cyclic shift on the polling list. In Refs. [3-6], the proposed polling schemes use a cyclic scheduling algorithm, where all stations are served sequentially according to their position on the polling list and when the last station is polled, the PC repeats the servicing cycle using the same order. In contrast to this cyclic scheduling algorithm, the proposed cyclic shift on the sequence of polled stations at the beginning of each PCF round (servicing cycle) spreads the packet rejections to all active stations in the network uniformly and provides more bandwidth for actual voice transmissions. Another important characteristic of the proposed polling scheme is the immediate removal of the silent stations from the polling list for a number of PCF rounds, based on the hangover duration of the used silence detectors. Refs. [5,6] considered the removal of silent stations from the polling list provided that these stations responded with NULL frames to a number of consecutive CF-Poll frames. In the CSSR polling scheme, the silent stations are immediately removed from the AP polling list and remain out of it for a number of PCF rounds. This procedure does not cause additional voice packet 


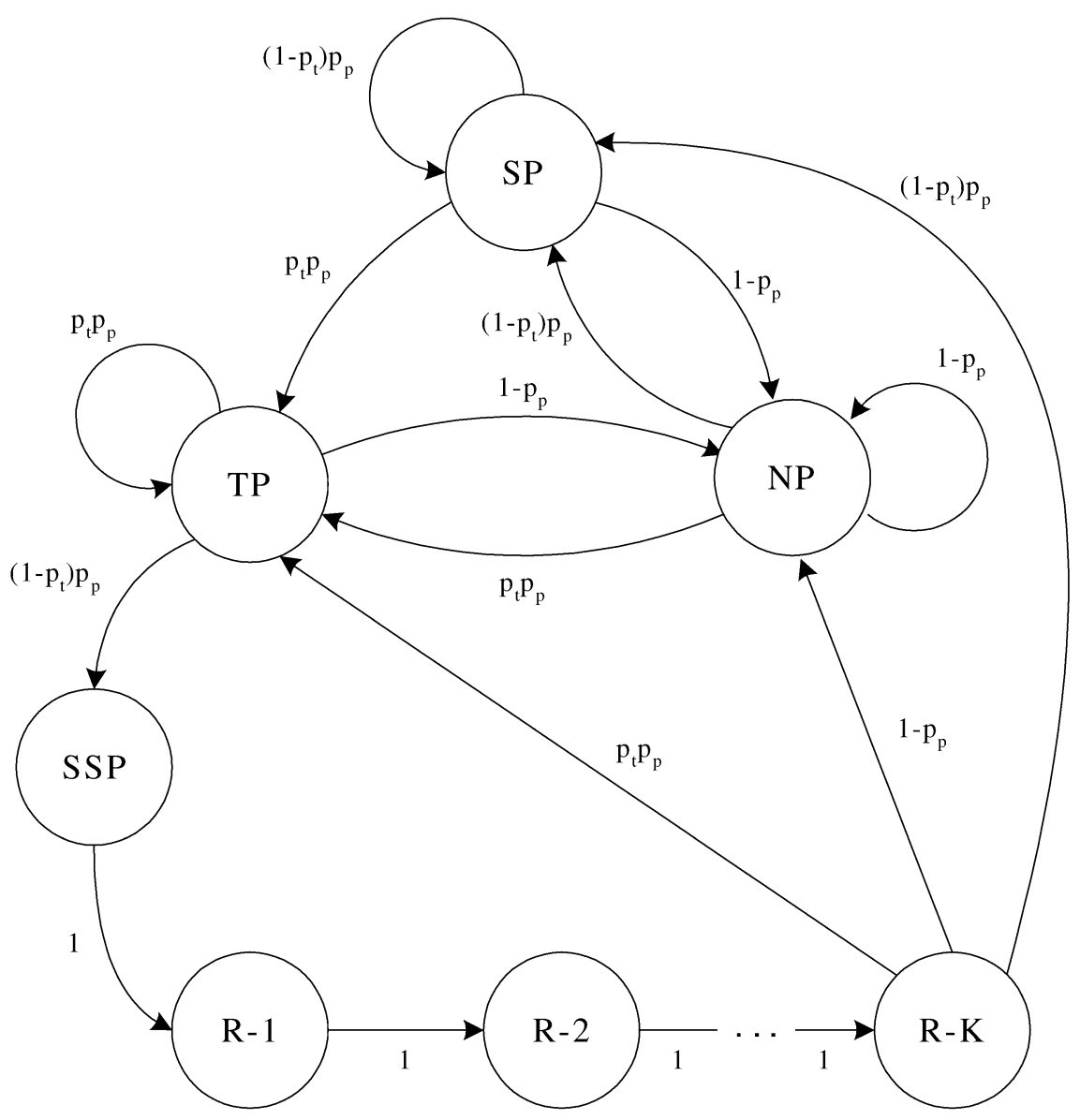

Fig. 4. The CSSR polling scheme: finite-state Markov chain for a voice station.

rejections, if the duration of a station removal is limited to the hangover duration, and further improves the PCF performance, since a silent state polled station, which otherwise must transmit a NULL frame, is replaced by a not polled station without any bandwidth loss. Moreover, the synchronization that forces the stations to generate voice packets at the same instant guarantees small and bounded delays. This dynamically adaptable scheme is called CSSR polling and is analyzed in the rest of this paper.

According to the CSSR polling scheme, a voice station is modeled as the discrete-time Markov chain, shown in Fig. 4. All state transitions occur at the end of the PCF round. During a CFP period, a voice station may be polled with probability $p_{\mathrm{p}}$ (its analytic expression is derived in Section 3 ) and is at one of the following states:

State SP. The station is in Silent-Polled (SP) state, when it is polled and has no voice packet to transmit.

State NP. The station leaves the SP state and enters the Not-Polled (NP) state, when polling cannot be completed and the station is among the not polled stations.

State TP. The station leaves the SP state and enters the Talk-Polled (TP) state, when a voice packet is generated (which happens with probability $p_{\mathrm{t}}$ ) and the PC polls the station. A voice station remains in the TP state if it is in talk spurt and is polled continuously. If the station is in talk spurt and the PC does not poll it, the station moves to the NP state.

State SSP. If the station's talk spurt ends and the PC polls the station, the station transmits a NULL frame and moves to the Start of Silence-Polled (SSP) state and remains in this state, until the next PCF round.

State $R-i$. When a silent period is detected, the PC removes the station from the active polling list for $K$ rounds and the station passes through the Removed-i (Ri) states. After $K$ rounds, the station is repositioned on the polling list and depending on its position in the polling list and the transmission of a voice or a NULL packet, the station returns either to the NP, TP or SP state.

Using this model, we can estimate an upper bound to the number of connections that can be accommodated by PCF for various values of $K$, while the condition $P_{\text {drop }}<0.01$ is satisfied, where $P_{\text {drop }}$ denotes the packet loss probability.

\section{Performance evaluation}

In our analysis, we initially consider a system that experiences no packet loss, the polling list is exhausted in 
one PCF round, all stations are polled once in each round and the stations that form a connection are polled successively, therefore dynamic polling and silence detection are not used. Let $T_{\mathrm{CFPR}}, T_{\mathrm{CFP}}$, and $T_{\mathrm{CP}}$ denote the duration of CFPR, CFP, and $\mathrm{CP}$ intervals, respectively. Then

$T_{\mathrm{CFPR}}=T_{\mathrm{CFP}}+T_{\mathrm{CP}}$

The values of $T_{\mathrm{CFP}}$ and $T_{\mathrm{CP}}$ may vary but their sum is always constant. For calculating the maximum number of conversations that can be accommodated by PCF, stations using the DCF mode are allowed to use a minimum bandwidth, which is defined by Ref. [1] as

$T_{\operatorname{min~} \mathrm{CP}}=T_{\max \mathrm{MPDU}}+2 \mathrm{SIFS}+2 a+8 T_{\mathrm{ACK}}+\mathrm{DIFS}$

where $a$ is the SlotTime parameter that a data station uses as time unit for updating its backoff counter, while $T_{\max }$ MPDU and $T_{\mathrm{ACK}}$ are the transmission duration of a maximum length data frame and an ACK frame, respectively.

The CFP shall be maximum, when the $\mathrm{CP}$ is minimum, since their sum is constant, but the CFP may be reduced due to the DCF traffic. Let $T_{\mathrm{FS}}$ be a random variable that describes the delay at the start of CFP, $T_{\mathrm{RTS}}$ and $T_{\mathrm{CTS}}$ are the transmission durations of an RTS and CTS frame, respectively. According to Ref. [1], the maximum value of this random variable is

$T_{\max \mathrm{FS}}=T_{\mathrm{RTS}}+T_{\mathrm{CTS}}+T_{\max \mathrm{MPDU}}+T_{\mathrm{ACK}}+3 \mathrm{SIFS}$

For calculating an upper bound of the number of conversations that the PC can handle, we consider that $T_{\mathrm{CFP}}^{\prime}=$ $T_{\max \text { CFP }}-T_{\max \text { FS }}$ is the time length of CFP and Eq. (1) is modified as:

$T_{\mathrm{CFPR}}=T_{\mathrm{CFP}}^{\prime}+T_{\min \mathrm{CP}}-a+T_{\max \mathrm{FS}}$

As in many papers in the literature [8-11], we also considered intra-BSS calls. This consideration is not the most realistic one, but it was done in order to concentrate on the analysis on the proposed mechanism and to be able to make comparisons with the results of these published works. Therefore, the duration $T_{\text {Con }}$ of voice packets exchange between stations belonging to the same connection according to Fig. 1 is given by

$T_{\text {Con }}=2\left(T_{\text {CF-Poll }}+T_{\mathrm{v}}+T_{\text {ACK }}+2\right.$ SIFS + PIFS $)$

where $T_{\mathrm{CF}-\mathrm{Poll}}$ is the transmission time of a CF-Poll frame and $T_{\mathrm{v}}$ is the transmission time of a voice packet. Let $N_{\mathrm{C} \max }$ denote the maximum number of stations that can be handled during PCF in case of CBR voice traffic, then

$T_{\text {CFP }}^{\prime}=$ PIFS $+T_{\text {Beacon }}+\frac{N_{\mathrm{C} \text { max }}}{2} T_{\text {Con }}+T_{\text {CF-END }}$

where $T_{\text {CF-END }}$ is the transmission time of a CF-END frame.

Using Eqs. (2)-(4), we can calculate the maximum number $N_{\mathrm{C} \max }$ of voice stations an IEEE802.11 BSS network can support for various values of CFPR interval, which is

$N_{\mathrm{C} \max }=$

$2\left\lfloor\frac{T_{\mathrm{CFPR}}-T_{\text {Beacon }}-\mathrm{SIFS}-T_{\max \text { FS }}-\text { PIFS }-T_{\text {CF-END }}-T_{\min \mathrm{CP}}}{T_{\mathrm{Con}}}\right\rfloor$

and $T_{\text {Beacon }}$ is the transmission time of a Beacon frame.

According to the IEEE $802.11 \mathrm{~b}$ standard, the physical header transmission rate is different from the transmission rate of the rest of the frame and an optional shorter physical header may be used, when maximum throughput is required. In this case, the physical header consists of two parts: the first part is $H_{\mathrm{PH} 1}$ bits long and its transmission rate is $R_{\mathrm{PH} 1}$, while the second part is $H_{\mathrm{PH} 2}$ bits long and its transmission rate is $R_{\mathrm{PH} 2}$. If $T_{\mathrm{S}}$ is the voice packet generation interval, $R_{\mathrm{S}}$ is the voice sampling rate, $H_{\mathrm{MAC}}$ is the number of bits of the MAC header-trailer, $T_{\mathrm{S}} R_{\mathrm{S}}$ bits is the size of the voice packet and the short physical header is used, the transmission time $T_{\mathrm{v}}$ of a voice frame is given by

$T_{\mathrm{v}}=\frac{H_{\mathrm{PH} 1}}{R_{\mathrm{PH} 1}}+\frac{H_{\mathrm{PH} 2}}{R_{\mathrm{PH} 2}}+\frac{H_{\mathrm{MAC}}+T_{\mathrm{CFPR}} R_{\mathrm{S}}}{R_{\mathrm{C}}}$

since $T_{\mathrm{S}}=T_{\mathrm{CFPR}}$.

\subsection{The voice packet drop probability}

In this subsection, an analytical approach is presented in order to derive an upper bound to the number of voice stations handled by PCF, when silence detection is employed. We have considered an ideal channel, the CSSR polling scheme and a voice station with the homogenous discrete-time Markov chain described in Section 2.2.

Let $P[X \mid Y]$ denotes the probability of a station going to state $X$ on the next PCF round, given that the station is at state $Y$ at the current round. Then, the state stationary transition probabilities are given by:

$$
\begin{aligned}
& P[\mathrm{TP} \mid \mathrm{TP}]=p_{\mathrm{p}} p_{\mathrm{t}}, \quad P[\mathrm{TP} \mid \mathrm{SP}]=p_{\mathrm{p}} p_{\mathrm{t}}, \\
& P[\mathrm{TP} \mid \mathrm{NP}]=p_{\mathrm{p}} p_{\mathrm{t}}, \quad P[\mathrm{TP} \mid R-K]=p_{\mathrm{p}} p_{\mathrm{t}}, \\
& P[\mathrm{SP} \mid \mathrm{SP}]=p_{\mathrm{p}}\left(1-p_{\mathrm{t}}\right), \quad P[\mathrm{SP} \mid \mathrm{NP}]=p_{\mathrm{p}}\left(1-p_{\mathrm{t}}\right), \\
& P[\mathrm{SP} \mid R-K]=p_{\mathrm{p}}\left(1-p_{\mathrm{t}}\right), \quad P[\mathrm{SSP} \mid \mathrm{TP}]=p_{\mathrm{p}}\left(1-p_{\mathrm{t}}\right), \\
& P[\mathrm{NP} \mid \mathrm{NP}]=1-p_{\mathrm{p}}, \quad P[\mathrm{NP} \mid \mathrm{TP}]=1-p_{\mathrm{p}}, \\
& P[\mathrm{NP} \mid \mathrm{SP}]=1-p_{\mathrm{p}}, \quad P[\mathrm{NP} \mid R-K]=1-p_{\mathrm{p}}, \\
& P[R-1 \mid \mathrm{SSP}]=1, \quad \\
& P[R-2 \mid R-1]=1, \ldots, P[R-K \mid R-(K-1)]=1
\end{aligned}
$$




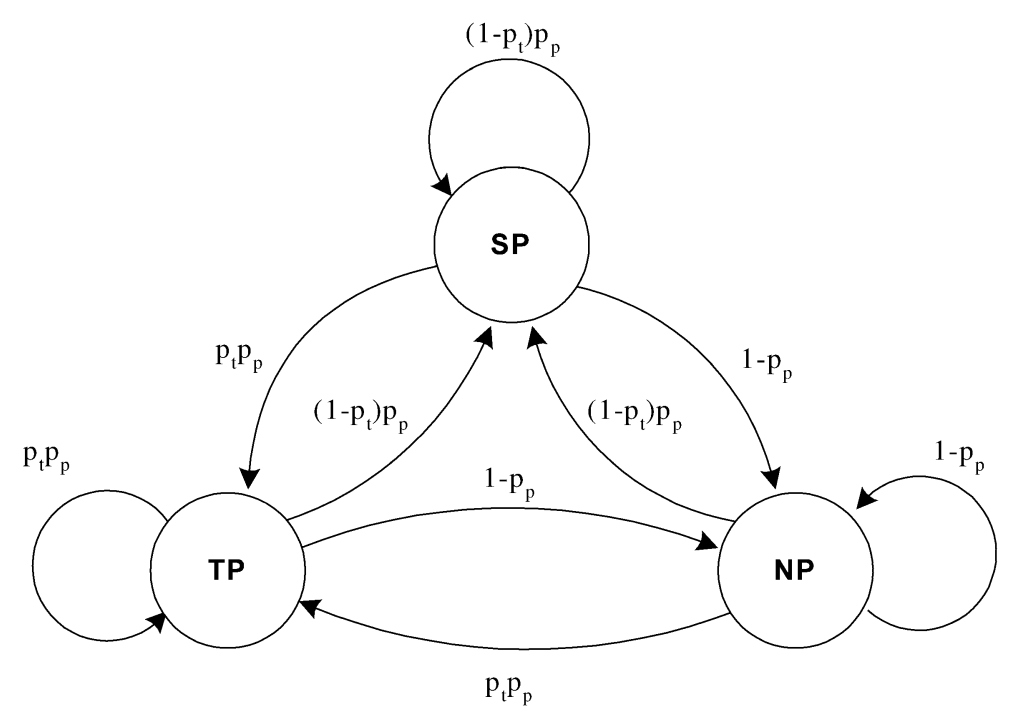

Fig. 5. The Cyclic Shift polling scheme: finite-state Markov chain for a voice station.

Let $\Pi_{X}$ be the steady state probability of a station being at state $X$. Then:

$$
\begin{aligned}
& \Pi_{R-1}=\Pi_{\mathrm{SSP}}, \quad \Pi_{R-K}=\Pi_{R-(K-1)}=\cdots=\Pi_{R-1} \\
& \Pi_{R}=K \Pi_{R-1}=K \Pi_{\mathrm{SSP}}
\end{aligned}
$$

where $\Pi_{R}$ is the steady state probability of a station being removed from polling list for $K$ PCF rounds, while $\Pi_{R-1}, \Pi_{R-2}, \ldots, \Pi_{R-K}$ are the steady state probabilities of a station being removed from the polling list for the 1st, 2nd, ..., Kth PCF round. Then:

$$
\begin{aligned}
& \Pi_{\mathrm{SSP}}=p_{\mathrm{p}}\left(1-p_{\mathrm{t}}\right) \Pi_{\mathrm{TP}} \\
& \Pi_{\mathrm{TP}}=\frac{p_{\mathrm{p}} p_{\mathrm{t}}}{1-p_{\mathrm{p}} p_{\mathrm{t}}}\left(\Pi_{\mathrm{SP}}+\Pi_{\mathrm{NP}}+\Pi_{R-K}\right) \\
& \Pi_{\mathrm{SP}}=\frac{p_{\mathrm{p}}\left(1-p_{\mathrm{t}}\right)}{1-p_{\mathrm{p}}\left(1-p_{\mathrm{t}}\right)}\left(\Pi_{\mathrm{NP}}+\Pi_{R-K}\right)
\end{aligned}
$$

According to the probability conservation law

$$
\Pi_{\mathrm{TP}}+\Pi_{\mathrm{SP}}+\Pi_{\mathrm{NP}}+\Pi_{\mathrm{SSP}}+\Pi_{R}=1
$$

and by using Eqs. (6) -(11), we can calculate the stationary distribution of the discrete Markov chain:

$$
\begin{aligned}
\Pi_{\mathrm{TP}} & =\frac{p_{\mathrm{p}} p_{\mathrm{t}}}{1+K p_{\mathrm{p}}^{2} p_{\mathrm{t}}\left(1-p_{\mathrm{t}}\right)}, \\
\Pi_{\mathrm{SP}} & =\frac{p_{\mathrm{p}}\left(1-p_{\mathrm{t}}\right)\left(1-p_{\mathrm{p}} p_{\mathrm{t}}\right)}{1+K p_{\mathrm{p}}^{2} p_{\mathrm{t}}\left(1-p_{\mathrm{t}}\right)}, \\
\Pi_{\mathrm{NP}} & =\frac{1-p_{\mathrm{p}}}{1+K p_{\mathrm{p}}^{2} p_{\mathrm{t}}\left(1-p_{\mathrm{t}}\right)}, \quad \Pi_{\mathrm{SSP}}=\frac{p_{\mathrm{p}}^{2} p_{\mathrm{t}}\left(1-p_{\mathrm{t}}\right)}{1+K p_{\mathrm{p}}^{2} p_{\mathrm{t}}\left(1-p_{\mathrm{t}}\right)} \\
\Pi_{R} & =\frac{K p_{\mathrm{p}}^{2} p_{\mathrm{t}}\left(1-p_{\mathrm{t}}\right)}{1+K p_{\mathrm{p}}^{2} p_{\mathrm{t}}\left(1-p_{\mathrm{t}}\right)}
\end{aligned}
$$

According to CSSR, if $K=i$, a voice station is not polled for $i$-consecutive PCF rounds, but its location in the polling list is shifted $i$-times. If $K=0$ (a polling scheme without station removal) the chain is simplified to the one depicted in Fig. 5.

Since the CFP interval has been selected so that the voice delay requirements are always satisfied, the only parameter that defines the PCF performance is the probability of lost packets $P_{\text {drop }}$. A voice station drops packets either when it is in talk spurt and at the NP state, or when it is at an (R-i) state and a talk spurt begins before the station is repositioned on the active polling list. When the voice station is in talk spurt and at the NP state, the probability of lost packets $P_{\text {drop1 }}$ is given by:

$P_{\mathrm{drop} 1}=\Pi_{\mathrm{NP}} p_{\mathrm{t}}$

Substituting Eqs. (12)-(14), we find that

$P_{\text {drop } 1}=\frac{\left(1-p_{\mathrm{p}}\right) p_{\mathrm{t}}}{1+K p_{\mathrm{p}}^{2} p_{\mathrm{t}}\left(1-p_{\mathrm{t}}\right)}$

Let $p_{\mathrm{st}}\left[t_{1} \leq D_{\mathrm{s}} \leq t_{2}\right]$ denotes the probability that the duration of a silent period $D_{\mathrm{s}}$ is between $t_{1}$ and $t_{2}$, which means that a talk spurt begins, when the station is at an (R-i) state. If the used silence detector has a hangover, which is $H$ times the CFPR interval, the silence gaps are greater than the hangover. So the minimum duration of a silent gap is $(H+1) T_{\mathrm{CFPR}}$. When a station is removed from the active polling list for $K$ PCF rounds, the time length that has passed from the detection of the transition to the SSP state is $(K+$ 1) $T_{\text {CFPR }}$ (including the CFPR interval that the station was at the SSP state). Further, since the silent periods of a voice call follow the exponential distribution with mean duration $d_{\mathrm{s}}$, the probability that a silent period terminates during 
the interval $\left[(H+1) T_{\mathrm{CFPR}},(K+1) T_{\mathrm{CFPR}}\right]$ is calculated as

$$
\begin{aligned}
& p_{\mathrm{st}}\left[(H+1) T_{\mathrm{CFPR}} \leq D_{\mathrm{s}} \leq(K+1) T_{\mathrm{CFPR}}\right] \\
& \quad=\int_{(H+1) T_{\mathrm{CPP}}}^{(K+1) T_{\mathrm{CPPR}}} \frac{1}{d_{\mathrm{s}}} \exp \left(-\frac{1}{d_{\mathrm{s}}} t\right) \mathrm{d} t \\
& \quad=\exp \left(-\frac{1}{d_{\mathrm{s}}}(H+1) T_{\mathrm{CFPR}}\right)-\exp \left(-\frac{1}{d_{\mathrm{s}}}(K+1) T_{\mathrm{CFPR}}\right)
\end{aligned}
$$

In this case, the probability $P_{\mathrm{drop} 2}$ of lost packets is given by:

$$
P_{\text {drop2 }}=
$$$$
\begin{cases}\Pi_{R} p_{\mathrm{st}}\left[(H+1) T_{\mathrm{CFPR}} \leq D_{\mathrm{s}} \leq(K+1) T_{\mathrm{CFPR}}\right] & \text { if } H<K, \\ 0, & \text { otherwise }\end{cases}
$$

that can be handled by PCF, when all served stations are in talk spurt.

According to the CSSR polling scheme, if during a CFP round the number of stations on the active polling list ( $N-$ $N_{\mathrm{r}}$ ) is less than $N_{\mathrm{t} \text { max }}$, then every station is polled. If $N-$ $N_{\mathrm{r}}>N_{\mathrm{t} \text { max }}$, only $N_{\mathrm{p}}$ stations are polled, the stations holding the first $N_{\mathrm{p}}$ positions on the active polling list, while the rest $\left(N-N_{\mathrm{r}}-N_{\mathrm{p}}\right)$ stations are not polled. $N_{\mathrm{p}}$ depends on the number of silent and talk stations, their distribution on the polling list and the maximum duration of CFP. Therefore, the conditional probability $p_{\mathrm{np}} \mid N_{\mathrm{r}}, N_{\mathrm{p}}$ that a station is not polled during a CFP is given by

$p_{\mathrm{np} \mid N_{\mathrm{r}}, N_{\mathrm{p}}}= \begin{cases}\frac{N-N_{\mathrm{r}}-N_{\mathrm{p}}}{N-N_{\mathrm{r}}} & \text { if } 0 \leq N_{\mathrm{r}} \leq N-N_{\mathrm{t} \max }-1, \\ 0 & \text { if } N-N_{\mathrm{t} \max } \leq N_{\mathrm{r}} \leq N\end{cases}$

Using Eqs. (13), (16) and (17), we have that

$P_{\text {drop } 2}= \begin{cases}\frac{K p_{\mathrm{p}}^{2} p_{\mathrm{t}}\left(1-p_{\mathrm{t}}\right)}{1+K p_{\mathrm{p}}^{2} p_{\mathrm{t}}\left(1-p_{\mathrm{t}}\right)}\left(\exp \left(-\frac{1}{d_{\mathrm{s}}}(H+1) T_{\mathrm{CFPR}}\right)-\exp \left(-\frac{1}{d_{\mathrm{s}}}(K+1) T_{\mathrm{CFPR}}\right)\right) & \text { if } H<K, \\ 0, & \text { otherwise }\end{cases}$

Therefore, the probability of lost packets $P_{\text {drop }}$ is derived by substituting Eqs. (15) and (18) to the following relation:

$P_{\text {drop }}=P_{\text {drop } 1}+P_{\text {drop2 }}$

\subsection{The basic system probabilities}

From the previous analysis, it is evident that we must know the probabilities $p_{\mathrm{t}}$ and $p_{\mathrm{p}}$ in order to calculate the voice packet loss probability $P_{\text {drop }}$. As defined is Section 2 , the probability $p_{\mathrm{t}}$ of a voice station to be in a talk spurt is given by

$p_{\mathrm{t}}=\frac{d_{\mathrm{t}}}{d_{\mathrm{t}}+d_{\mathrm{s}}}$

So it remains to find the probability $p_{\mathrm{p}}$ of a station to be polled. We will initially calculate the probability $p_{\text {np }}$ that a station is not to be polled, and probability $p_{\mathrm{p}}$ will be derived by:

$p_{\mathrm{p}}=1-p_{\mathrm{np}}$

The probability $p_{\mathrm{np}}$ depends on the total number of voice stations, the number of stations not placed on the active polling list (they are in silent state) and the number of stations that are polled during a PCF round. A number of stations at the end of the active polling list may not be served in a PCF round, if the total service time for idle and talk stations exceeds CFPmaxDuration. Let $N$ be the number of voice stations that form the main polling list, $N_{\mathrm{r}}$ the number of stations that do not participate in the active polling list during a PCF round and $N_{\mathrm{p}}$ the number of stations polled during a PCF round. Additionally, we denote with $N_{\mathrm{t} \text { max }}$ the maximum number of stations where $N_{\mathrm{r}}$ is the number of stations removed from the active polling list and $N_{\mathrm{p}}$ is the number of polled stations. In Eq. (22), we observe that the conditional probability $p_{\mathrm{np} \mid N_{\mathrm{r}}, N_{\mathrm{p}}}$ must be calculated only for $0 \leq N_{\mathrm{r}} \leq N-N_{\mathrm{t} \text { max }}-1$, since it is zero for all other values of $N_{\mathrm{r}}$.

For a given number $N_{\mathrm{r}}$, the number of stations that can be polled has a maximum value $N_{\mathrm{p} \text { max }}$ depending on the length of CFP. Thus, for some combinations of the number of stations in talk spurt and the number of stations in silent, the PC polls $N_{\mathrm{p} \text { max }}$ stations or $\left(N-N_{\mathrm{r}}\right)$ stations, whatever of the two events happens first. Finding the probability $P_{N_{\mathrm{p}} \mid N_{\mathrm{r}}}$ that the PC polls $N_{\mathrm{p}}$ stations given that it has removed $N_{\mathrm{r}}$ stations and the probability $P_{N_{\mathrm{r}}}$ that the PC has removed $N_{\mathrm{r}}$ stations, then from the total probability theorem and Eq. (22), we have that:

$p_{\mathrm{np}}=\sum_{N_{\mathrm{r}}=0}^{N-N_{\mathrm{t} \max }-1} \sum_{N_{\mathrm{p}}=N_{\mathrm{t}} \max }^{\min \left(N_{\mathrm{p}} \max , N-N_{\mathrm{r}}\right)} \frac{N-N_{\mathrm{r}}-N_{\mathrm{p}}}{N-N_{\mathrm{r}}} P_{N_{\mathrm{p}} \mid N_{\mathrm{r}}} P_{N_{\mathrm{r}}}$

Now, we must relate parameters $N_{\mathrm{t} \max }, N_{\mathrm{p} \max }$ to the probabilities $P_{N_{\mathrm{r}}}$ and $P_{N_{\mathrm{p}} \mid N_{\mathrm{r}}}$. The duration $T_{\mathrm{t}}$ of a voice packet transmission and the duration $T_{\mathrm{s}}$ of a NULL frame transmission are given by

$T_{\mathrm{t}}=T_{\text {CF-Poll }}+\mathrm{SIFS}+T_{\mathrm{v}}+\mathrm{SIFS}+T_{\mathrm{ACK}}+\mathrm{PIFS}$

$T_{\mathrm{s}}=T_{\mathrm{CF}-\mathrm{Poll}}+\mathrm{SIFS}+T_{\mathrm{NULL}}+\mathrm{SIFS}$

where NULL bits is the size of a NULL frame including physical and MAC headers, and

$T_{\mathrm{NULL}}=\frac{H_{\mathrm{PH} 1}}{R_{\mathrm{PH} 1}}+\frac{H_{\mathrm{PH} 2}}{R_{\mathrm{PH} 2}}+\frac{\mathrm{NULL}}{R_{\mathrm{C}}}$ 


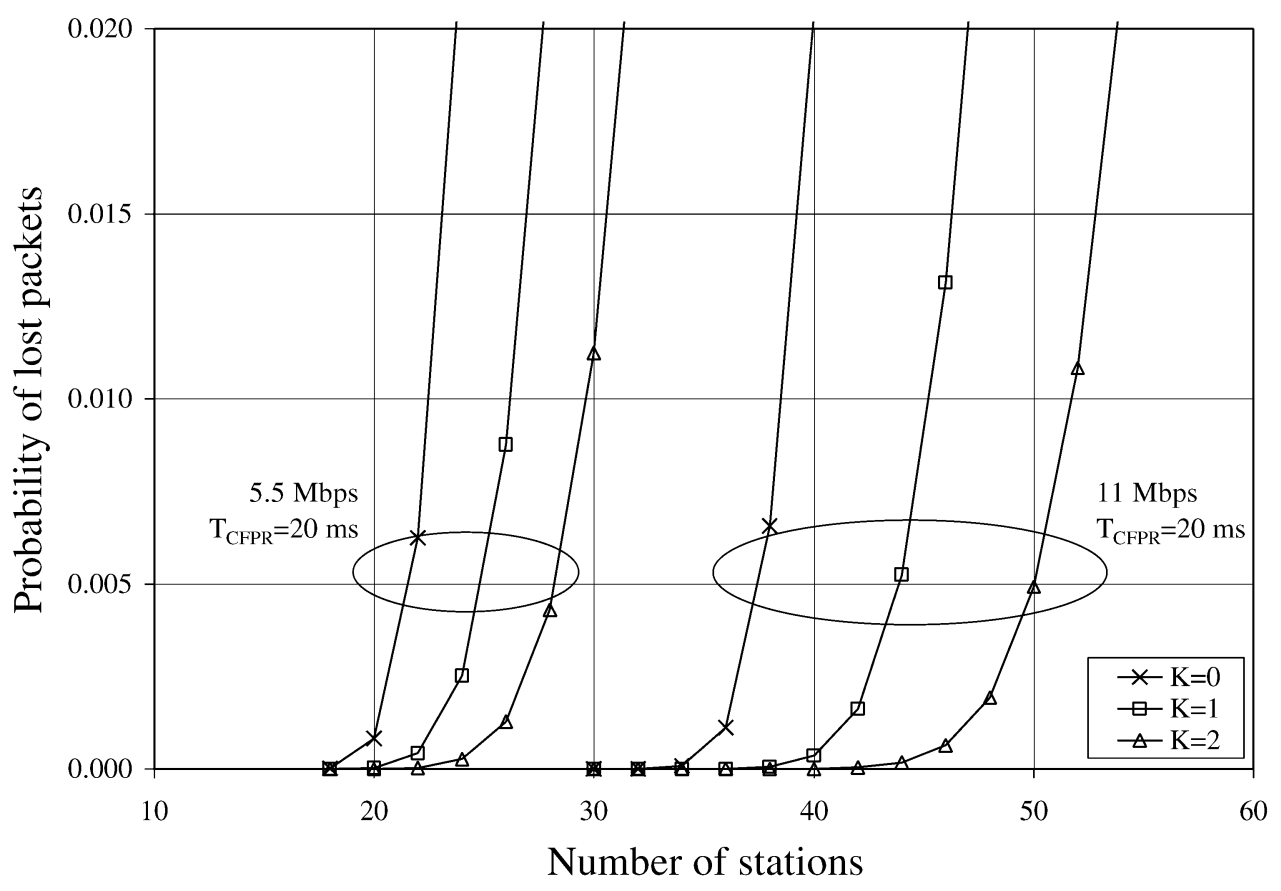

Fig. 6. Probability of lost packets versus the number of stations for $H \geq K$.

The maximum number $N_{\mathrm{t} \max }$ of stations that the PC can poll, when all of them are in talk spurt, depends on the maximum duration of the CFP and the voice packet transmission duration $T_{\mathrm{t}}$ and can be found by:

$$
\begin{aligned}
& N_{\mathrm{t} \text { max }}= \\
& \left\lfloor\frac{T_{\mathrm{CFPR}}-T_{\text {Beacon }}-\mathrm{SIFS}-T_{\max \text { FS }}-\mathrm{PIFS}-T_{\mathrm{CF}-\mathrm{END}}-T_{\min \mathrm{CP}}}{T_{\mathrm{t}}}\right\rfloor
\end{aligned}
$$

We define as $N_{\mathrm{p} \text { max }}$ the maximum number of stations that can be polled, when all the sequentially served stations are in silent mode. $N_{\mathrm{p} \text { max }}$ is calculated by considering that $T_{\mathrm{t}}>$ $T_{\mathrm{s}}$. Therefore, depending on the ratio $T_{\mathrm{t}} / T_{\mathrm{s}}$, the transmission time of a station in talk state can be replaced by the transmission time of one or more silent stations. Since $N_{\mathrm{t} \text { max }}$ is the maximum number of polled stations, when all are in talk state, the maximum number of stations that can be polled, when all are in silent mode, is $N_{\mathrm{t} \text { max }} T_{\mathrm{t}} / T_{\mathrm{s}}$. Since the PC serves sequentially the stations on the active list and does not know if the next station on the list is in talk or in silent state, the above $N_{\mathrm{t} \text { max }}$ has to be decreased by one, for satisfying the requirement that the duration of CFP does not exceed its maximum value. Therefore, $N_{\mathrm{p} \text { max }}$ is given by:

$N_{\mathrm{p} \max }=\left\lfloor\left(N_{\mathrm{t} \max }-1\right) \frac{T_{\mathrm{t}}}{T_{\mathrm{s}}}+1\right\rfloor$

Let $p_{\mathrm{r}}$ the probability a station is removed from the polling list. Then, the $N_{\mathrm{r}}$ stations during a PCF round have a binomial mass function and

$P_{N_{\mathrm{r}}}=\left(\begin{array}{c}N \\ N_{\mathrm{r}}\end{array}\right) p_{\mathrm{r}}^{N_{\mathrm{r}}}\left(1-p_{\mathrm{r}}\right)^{N-N_{\mathrm{r}}}$

The probability $p_{\mathrm{r}}$ can be calculated by Eq. (13), where the probability $p_{\mathrm{p}}$ is substituted by $N_{\mathrm{p}} /\left(N-N_{\mathrm{r}}\right)$, which indicates that a station could be at one of the $N_{\mathrm{p}}$ first positions of $N-N_{\mathrm{r}}$ stations on the polling list. So

$p_{\mathrm{r}}=\frac{K\left(\frac{N_{\mathrm{p}}}{N-N_{\mathrm{r}}}\right)^{2} p_{\mathrm{t}}\left(1-p_{\mathrm{t}}\right)}{1+K\left(\frac{N_{\mathrm{p}}}{N-N_{\mathrm{r}}}\right)^{2} p_{\mathrm{t}}\left(1-p_{\mathrm{t}}\right)}$,

$0 \leq N_{\mathrm{r}} \leq N-N_{\mathrm{t} \max }-1$

Finally, the probability $P_{N_{\mathrm{p}} \mid N_{\mathrm{r}}}$ is defined by the number of stations that are in talk spurt, $N_{\mathrm{t}}$, and the number of stations that are in silent period during a CFP, $N_{\mathrm{p}}-N_{\mathrm{t}}$. The combinations of $N_{\mathrm{t}}$ and $N_{\mathrm{p}}-N_{\mathrm{t}}$ are constrained by the maximum number $N_{\mathrm{t} \text { max }}$ of stations in talk spurt that can be polled during a CFP. For this reason, we use the function $f(x)$ :

$f(x)= \begin{cases}1 & \text { if } x=0 \\ 0 & \text { if } x \neq 0\end{cases}$

which gives the valid combinations of $N_{\mathrm{t}}$ and $N_{\mathrm{p}}-N_{\mathrm{t}}$ for $x=\left\lceil\left(\left(N_{\mathrm{p}}-N_{\mathrm{t}}\right) T_{\mathrm{s}}\right) / T_{\mathrm{t}}\right\rceil+N_{\mathrm{t}}-N_{\mathrm{t} \text { max }}$. For example, if $N_{\mathrm{t} \max }=4, T_{\mathrm{t}}=2 T_{\mathrm{s}}$ and $N_{\mathrm{p}}=5$ then if $\left(N_{\mathrm{t}}, N_{\mathrm{p}}-N_{\mathrm{t}}\right)$ depicts the pair (number of polled stations in talk spurt, number of polled stations in silent state) during a CFP, the 


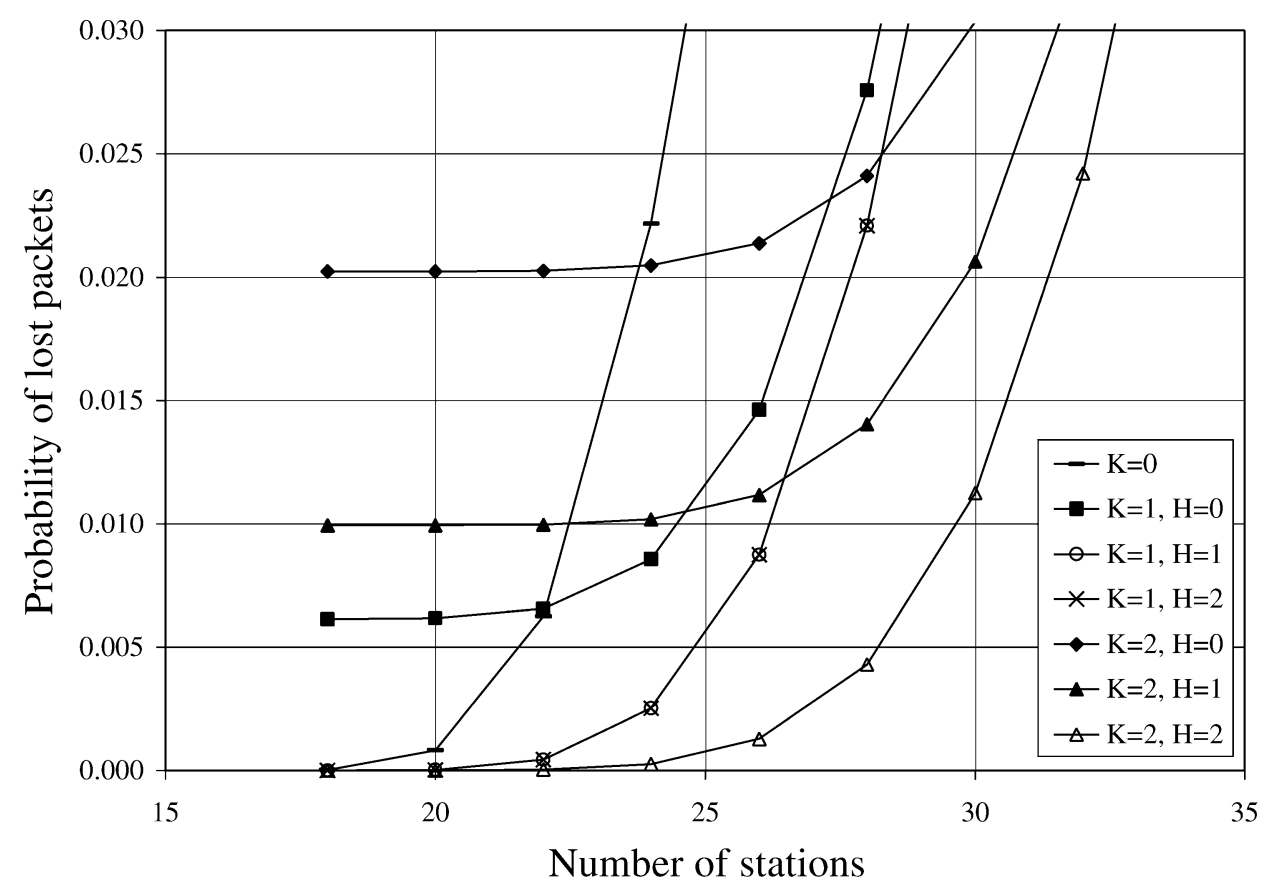

Fig. 7. The dependence of lost packets probability on $H$ and $K$.

pairs $(2,3),(3,2)$ are valid, while the pairs $(0,5),(1,4),(4,1)$, and $(5,0)$ are not valid.

A valid pair can form $\left(\frac{N_{\mathrm{p}}}{N_{\mathrm{t}}}\right)$ different combinations of talk and silent stations. These combinations may become fewer depending on the order of the talk and silent stations, since the PC polls the stations successively, estimates the remaining duration of the CFP in accordance to the previous polled stations and does not know if the next station on the list is in talk or silent state. Thus, to the previous example for the pair $(3,2)$, which gives 10 different orders of talk and silent stations, the combination (1st station talks, 2nd station talks, 3rd station silent, 4th station talks, 5th station silent) must be rejected, since the PC can handle the 5th station only if it is in silent state, but as the PC does not know the 5th station state, it ends the CFP after polling the 4th station. The rejected combinations are defined by the following function since we assume that a station is in talk state independently of all other stations with probability $p_{\mathrm{t}}$ and so the random variable $N_{\mathrm{t}}$ has the binomial mass function [20]. Substituting Eqs. (24)-(28) to Eq. (23), we find the probability $p_{\text {np }}$ and by using Eq. (16) we can calculate the probability $p_{\mathrm{p}}$.

\subsection{The effect of transmission errors on the CSSR polling scheme}

In this section, a burst error model is introduced for studying the total packet loss probability and the maximum number of supported voice stations. This model represents the fading conditions of the wireless medium and is based on a two-state discrete-time Markov chain [4,5,7]. The two states are called $G$ (good) and $B$ (bad) and indicate that the medium operates either at a low bit error rate (denoted by $\mathrm{BER}_{G}$ ) or at fading conditions with a higher error rate

$g\left(N_{\mathrm{p}}, N_{\mathrm{t}}, N_{\mathrm{t} \max }\right)=$

$\begin{cases}0 & \text { if } N_{\mathrm{p}}=N_{\mathrm{t} \text { max }} \vee N_{\mathrm{t}}=0 \vee N_{\mathrm{t}}+N_{\mathrm{p}}-N_{\mathrm{t} \text { max }}<N_{\mathrm{t} \max } \vee\left(\left(N_{\mathrm{t}}+N_{\mathrm{p}}-N_{\mathrm{t} \max }=N_{\mathrm{t} \max }\right) \wedge\left(\left(N_{\mathrm{t}}+1\right) T_{\mathrm{t}}+\left(N_{\mathrm{p}}-N_{\mathrm{t}}-1\right) T_{\mathrm{s}} \leq N_{\mathrm{t} \text { max }} T_{\mathrm{t}}\right)\right), \\ 1 & \text { if } N_{\mathrm{t}}+N_{\mathrm{p}}-N_{\mathrm{t} \text { max }}>N_{\mathrm{t} \text { max }} \vee\left(\left(N_{\mathrm{t}}+N_{\mathrm{p}}-N_{\mathrm{t} \text { max }}=N_{\mathrm{t} \max }\right) \wedge\left(\left(N_{\mathrm{t}}+1\right) T_{\mathrm{t}}+\left(N_{\mathrm{p}}-N_{\mathrm{t}}-1\right) T_{\mathrm{s}}>N_{\mathrm{t} \text { max }} T_{\mathrm{t}}\right)\right)\end{cases}$

Therefore, the probability that the number of polled stations during a PCF round is $N_{\mathrm{p}}$, is given by

$$
\begin{array}{r}
P_{N_{\mathrm{p}} \mid N_{\mathrm{r}}}=\sum_{N_{\mathrm{t}}=0}^{N_{\mathrm{t} \max }}\left[\left(\begin{array}{c}
N_{\mathrm{p}} \\
N_{\mathrm{t}}
\end{array}\right)-g\left(N_{\mathrm{p}}, N_{\mathrm{t}}, N_{\mathrm{t} \max }\right)\left(\begin{array}{c}
N_{\mathrm{p}}-1 \\
N_{\mathrm{t}}
\end{array}\right)\right] \\
\times p_{\mathrm{t}}^{N_{\mathrm{t}}}\left(1-p_{\mathrm{t}}\right)^{N_{\mathrm{p}}-N_{\mathrm{t}}} f\left(\left[\frac{\left(N_{\mathrm{p}}-N_{\mathrm{t}}\right) T_{\mathrm{s}}}{T_{\mathrm{t}}}\right]+N_{\mathrm{t}}-N_{\mathrm{t} \max }\right)
\end{array}
$$

(denoted by $\mathrm{BER}_{B}$ ). State $G$ changes to state $B$ with transition rate $\alpha$, while state $B$ changes to state $G$ with transition rate $\beta$. Let $p_{G}$ and $p_{B}$ denote the probabilities that the channel is in the $G$ or the $B$ state, respectively. Then

$p_{G}=\frac{\beta}{\alpha+\beta}, \quad p_{B}=\frac{\alpha}{\alpha+\beta}$

During a voice packet transmission time $T_{\mathrm{v}}$, the channel behaves according to one of the following cases: 


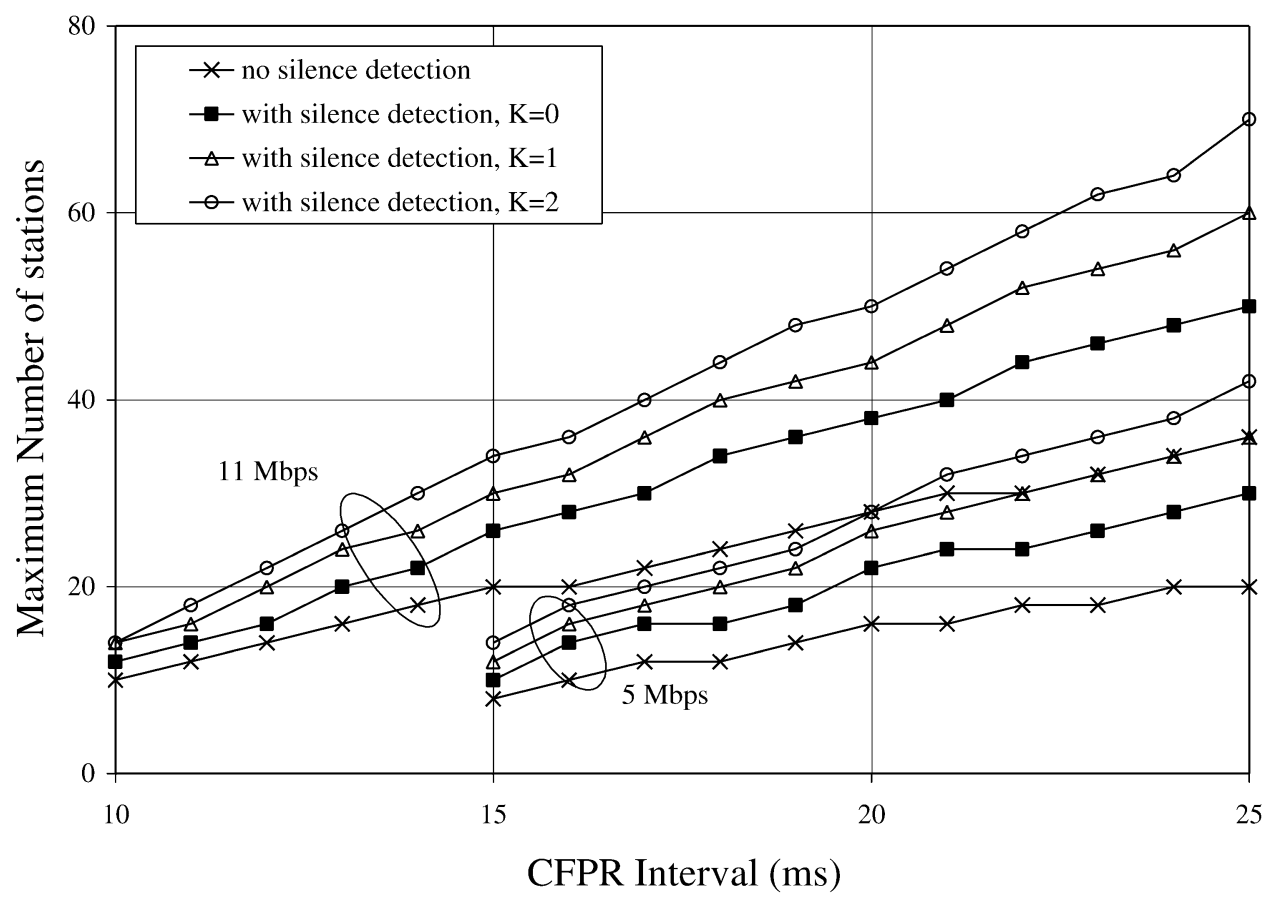

Fig. 8. The effect of CSSR to the maximum number of supported voice stations, when short physical headers are used.

Case 1. Always in $G$ state with probability:

$P_{\text {Case } 1}=p_{G} P\left(G>T_{\mathrm{v}}\right)=\frac{\beta}{\alpha+\beta} \mathrm{e}^{-\alpha T_{\mathrm{v}}}$

Case 2. Always in $B$ state with probability:

$P_{\text {Case } 2}=p_{B} P\left(B>T_{\mathrm{v}}\right)=\frac{\alpha}{\alpha+\beta} \mathrm{e}^{-\beta T_{\mathrm{v}}}$
Case 3. Undergoes one or more transitions between $G$ and $B$ states with probability:

$P_{\text {Case3 }}=1-P_{\text {Case1 }}-P_{\text {Case2 }}$

Therefore, the probabilities that a voice packet of $\left(H_{\mathrm{PH} 1}+\right.$ $\left.H_{\mathrm{PH} 2}+H_{\mathrm{MAC}}+T_{\mathrm{CFPR}} R_{\mathrm{S}}\right)$ bits is received with errors are

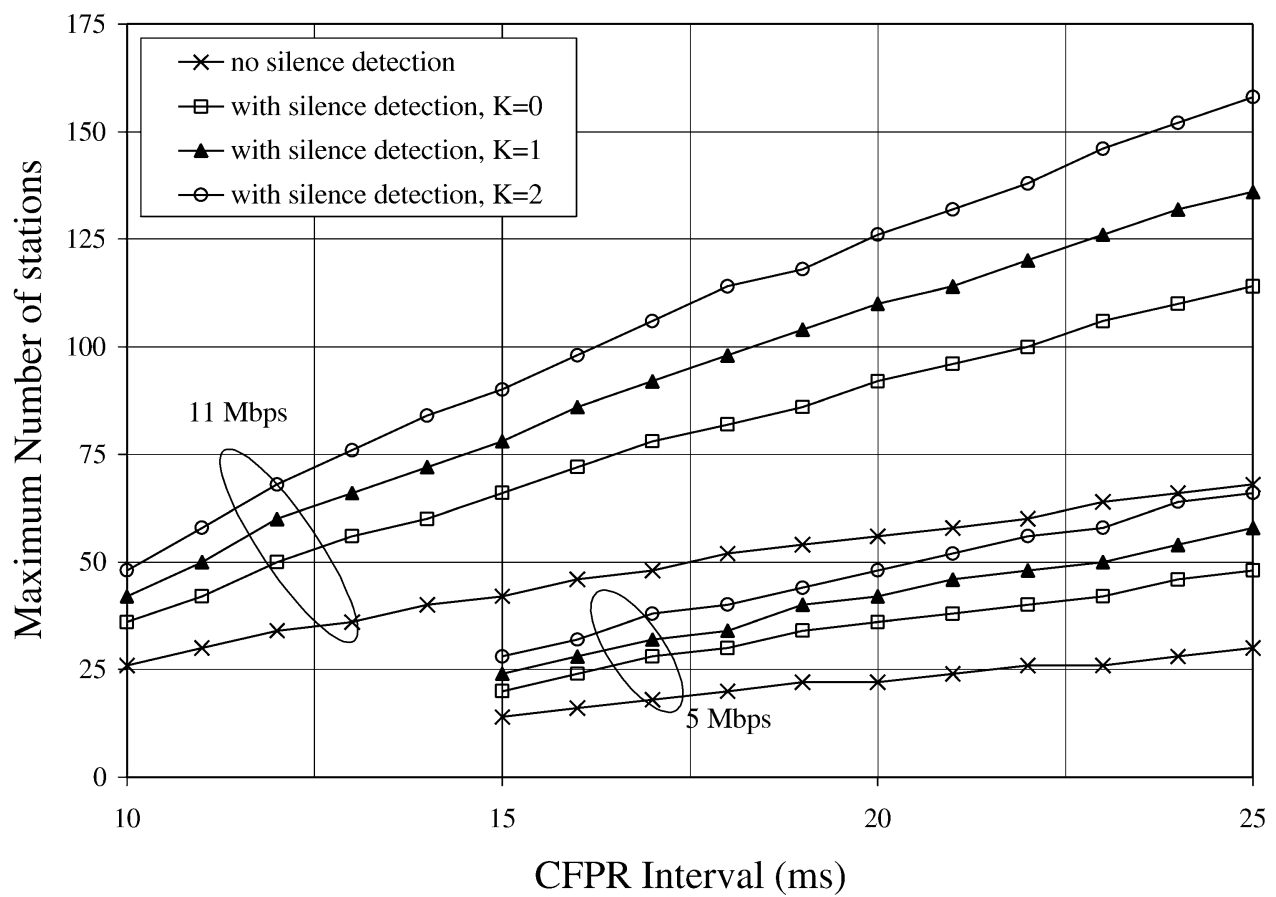

Fig. 9. The effect of CSSR to the maximum number of supported voice stations, when the physical headers are transmitted at the same rate as the payload. 


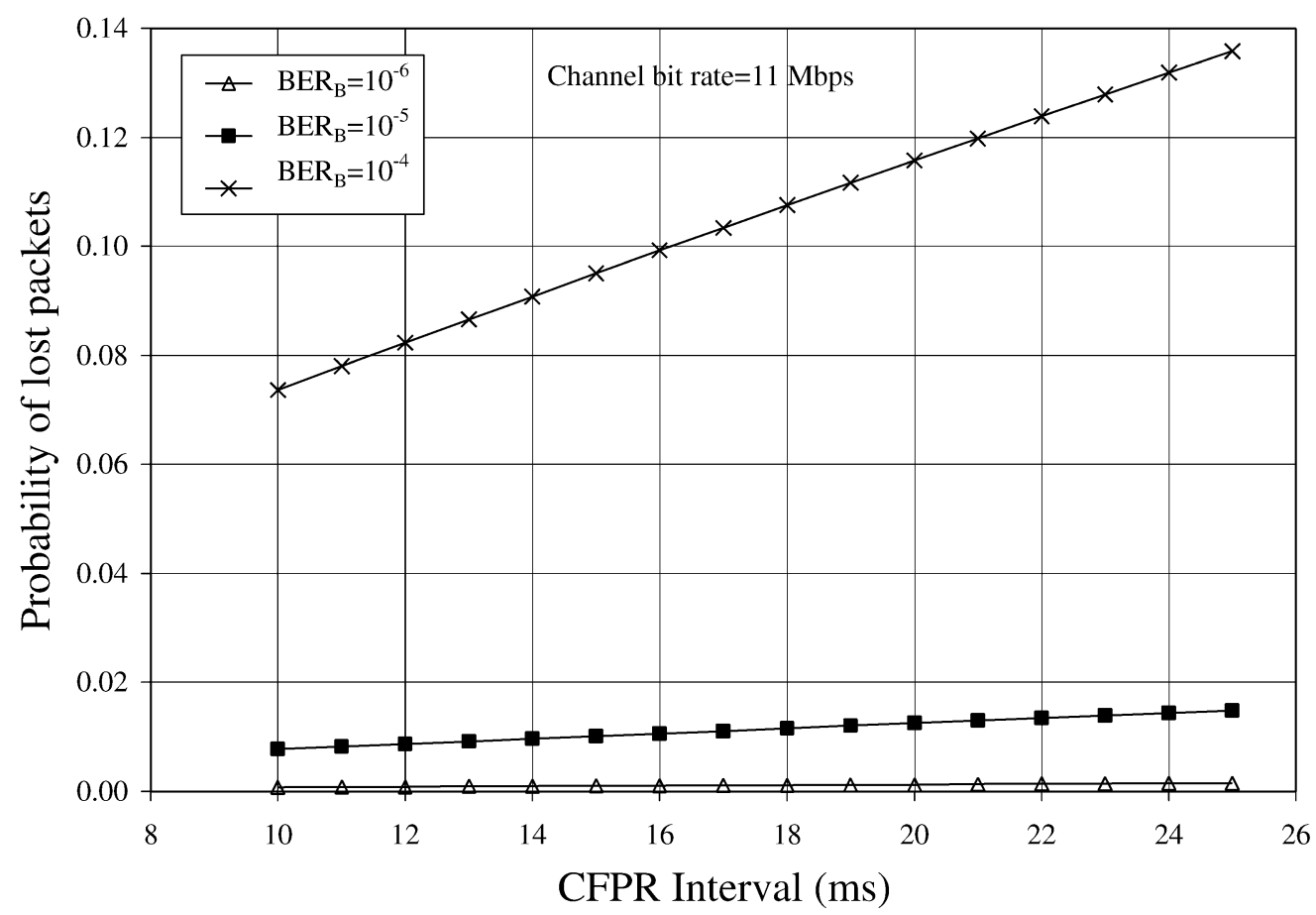

Fig. 10. Probability of packet errors versus the CFPR interval, when short physical headers are used.

given by:

$P_{\text {err_Case } 1}=1-\left(1-\mathrm{BER}_{G}\right)^{\left(H_{\mathrm{PH} 1}+H_{\mathrm{PH} 2}+H_{\mathrm{MAC}}+T_{\mathrm{CFPR}} R_{\mathrm{S}}\right)}$

$P_{\text {err_Case } 2}=1-\left(1-\mathrm{BER}_{B}\right)^{\left(H_{\mathrm{PH} 1}+H_{\mathrm{PH} 2}+H_{\mathrm{MAC}}+T_{\mathrm{CFPR}} R_{\mathrm{S}}\right)}$

$P_{\text {err_Case3 }} \leq P_{\text {err_Case2 }}$

Using Eqs. (29)-(34), we can approximate the total packet error probability by

$P_{\text {err }} \leq P_{\text {Case } 1} P_{\text {err_Case } 1}+P_{\text {Case } 2} P_{\text {err_Case2 }}+P_{\text {Case } 3} P_{\text {err_Case2 }}$

Therefore, the total lost packet probability, due to transmission errors and the CSSR polling scheme, can be upper bounded by substituting Eqs. (19) and (35) to the following relationship:

$P_{\text {total_drop }} \leq P_{\text {err }}+P_{\text {drop }}$

Table 1

The effect of errors on the supported number of voice stations for $K=1$ and using short physical headers

\begin{tabular}{|c|c|c|c|c|c|c|}
\hline \multirow[t]{3}{*}{ CFPR interval (ms) } & \multicolumn{3}{|c|}{ Channel bit rate $5.5 \mathrm{Mbps}$} & \multicolumn{3}{|c|}{ Channel bit rate $11 \mathrm{Mbps}$} \\
\hline & \multicolumn{3}{|l|}{$\mathrm{BER}_{B}$} & \multicolumn{3}{|l|}{$\mathrm{BER}_{B}$} \\
\hline & Ideal channel & $10^{-6}$ & $10^{-5}$ & Ideal channel & $10^{-6}$ & $10^{-5}$ \\
\hline 10 & & & & 14 & 12 & 12 \\
\hline 11 & & & & 16 & 16 & 14 \\
\hline 12 & & & & 20 & 20 & 18 \\
\hline 13 & & & & 24 & 24 & 20 \\
\hline 14 & & & & 26 & 26 & 22 \\
\hline 15 & 12 & 12 & 8 & 30 & 30 & - \\
\hline 16 & 16 & 16 & 10 & 32 & 32 & - \\
\hline 17 & 18 & 18 & 12 & 36 & 36 & - \\
\hline 18 & 20 & 20 & 12 & 40 & 38 & - \\
\hline 19 & 22 & 22 & 14 & 42 & 42 & - \\
\hline 20 & 26 & 24 & - & 44 & 44 & - \\
\hline 21 & 28 & 28 & - & 48 & 48 & - \\
\hline 22 & 30 & 28 & - & 52 & 50 & - \\
\hline 23 & 32 & 30 & - & 54 & 54 & - \\
\hline 24 & 34 & 34 & - & 56 & 56 & - \\
\hline 25 & 36 & 36 & - & 60 & 60 & - \\
\hline
\end{tabular}


The maximum number of voice stations supported by PCF is determined by limiting the total probability of lost packets to 0.01 .

\section{Numerical results}

In this section, we present and discuss numerical results showing the performance of the IEEE802.11 PCF procedure, when the CSSR polling scheme is used. The results of our analysis are derived considering that: MAC header $=34 \times 8$ bits (including the FCS field), Physical header first part $=9 \times 8$ bits, Physical header second part $=6 \times 8$ bits, $\max$ Payload $=2312 \times 8$ bits, $\mathrm{ACK}=30 \times 8$ bits, $\mathrm{RTS}=36 \times 8$ bits, $\mathrm{CTS}=30 \times 8$ bits, $\quad$ CF-Poll $=50 \times 8$ bits, $\quad$ CF-END $=36 \times 8$ bits, Beacon $=106 \times 8$ bits, NULL $=50 \times 8$ bits, SIFS $=10$ $\mu \mathrm{s}, \quad \mathrm{PIFS}=30 \mu \mathrm{s}, \quad$ DIFS $=50 \mu \mathrm{s}, \quad$ SlotTime $=20 \mu \mathrm{s}$, Physical header bit rates $=1 \mathrm{Mbps}$ (first part) and $2 \mathrm{Mbps}$ (second part), MAC header and payload bit rate $=5.5$ and $11 \mathrm{Mbps}, \mathrm{BER}_{G}=10^{-10}, \mathrm{BER}_{B}=10^{-6}, 10^{-5}$ and $10^{-4}$, $\alpha=30 \mathrm{~s}^{-1}$ and $\beta=10 \mathrm{~s}^{-1}$.

Initially, we consider an ideal channel in order to evaluate the performance of the CSSR polling scheme. Fig. 6 shows the dependence of the probability of lost packets to the number of voice stations accommodated by PCF, when the hangover $H$ is greater than or equal to the number $K$ of PCF rounds that a station is removed from the polling list. We notice that the number of supported conversations increases as the number $K$ increases, since NULL transmissions are replaced with voice packets. When $H \geq$ $K\left(P_{\text {drop } 1} \neq 0\right)$, the minimum duration of a station's silent period is greater than or equal to the duration of the station removal and thus the lost packets are caused only when a station is not polled and is in a talk spurt $\left(P_{\text {drop2 }}=0\right)$.

The effect of $H$ and $K$ to the probability of lost packets is depicted in Fig. 7. When $K=0$, the PC employs the Cyclic Shift polling scheme and a station rejects a packet if it is in a talk spurt and is not polled. For $K>0$, the PC uses the CSSR polling scheme. In this case, when $H<K$ the probability of lost packets grows up, since a station may drop a packet if a talk spurt begins before the station has been recalled to the active polling list $\left(P_{\text {drop2 }}>0\right)$ except, when the station is not polled and is in a talk spurt. So the CSSR polling scheme is more efficient when $H \geq K$ and therefore, the choice of the optimum value of parameter $K$ depends on the used hangover of the silence detector. When $H>K$ the method's performance does not increase (compare cases $K=1, H=1$ and $K=1, H=2$ in Fig. 7), since the method's advantages have been fully exploited when $H=K$.

Fig. 8 presents the maximum number of stations that can be managed by PCF (satisfying the condition $P_{\text {drop }}<0.01$ ) with and without silence detection and with the assumption of a hangover close to the duration of the temporary station removal from the active polling list. We notice that by determining the $K$ parameter properly, the proposed polling scheme improves significantly the PCF performance. Since in other papers, the numerical results are presented with the whole frame transmitted at the same rate, we also include Fig. 9 that presents the maximum number of stations achieved in this case. We observe that the transmission rate of the physical header has high impact on the IEEE802.11 WLANs throughput.

Finally, error conditions on the wireless medium worsen the PCF performance, as it is shown in Fig. 10. The packet error probability increases as the CFPR interval duration increases, since the voice packet length also increases. When the packet rejection rate exceeds $1 \%$ (as for $\mathrm{BER}_{B}=10^{-4}$ ), this results to unacceptable voice quality. The maximum number of voice stations supported by PCF with the implementation of the CSSR procedure for various fading mediums is shown in Table 1 . The cells with a hyphen denote that the requirement $P_{\text {total_drop }} \leq 0.01$ cannot be achieved under these conditions.

\section{Conclusions}

In this paper, we proposed the CSSR polling scheme that can be used along with silence detection on IEEE802.11 Wireless LANs for increasing the number of supported voice communications. The proposed scheme was analyzed and we calculated the maximum number of voice stations the PCF can accommodate as a function of the CFPR interval by using constant bit rate digitized voice and by guaranteeing a minimum bandwidth available for DCF. In case of using silence detection at the mobile terminals, the CSSR scheme can be used to support a higher number of voice conversations. The CSSR scheme changes the service sequence during each service cycle (PCF round) by using a dynamic cyclic shift mechanism. The exploitation of the silence detector's hangover for removing the idle stations from the polling list temporarily, improves the system performance without increasing the packet loss rate. The cyclic shift improves the fairness of the system and the removal of idle stations increases the number of supported stations. In comparison with other approaches, our proposed polling scheme provides higher number of voice stations and much lower packet delays, imposes simple implementation complexity and does not require any modification on the mobile terminals access mechanism. The next step of this work is to evaluate the use of CSSR polling scheme in IEEE802.11 networks interconnected via a backbone network and to determine end-to-end delay distributions for exchanging voice traffic.

\section{References}

[1] IEEE Std 802.11, Part 11: Wireless LAN Medium Access Control (MAC) and Physical Layer (PHY) Specifications, IEEE, 1999. 
[2] IEEE Std 802.11e/D1.3, Draft Supplement to IEEE Std 802.11: Medium Access Control (MAC) Enhancements for Quality of Service (QoS), IEEE, 2001, October.

[3] M. Visser, M. Zarki, Voice and data transmission over an 802.11 wireless network, Proceedings of IEEE PIMRC (1995) 648-652.

[4] B. Crow, I. Widjaja, J.G. Kim, P. Sakai, Investigation of the IEEE 802.11 medium access control (MAC) sublayer functions, Proceedings of INFOCOM (1997) 26-33.

[5] B. Crow, I. Widjaja, J.G. Kim, P. Sakai, IEEE 802.11 wireless local area networks, IEEE Communications Magazine 35 (9) (1997) $116-126$.

[6] W.M. Moh, D. Yao, K. Makki, Analyzing the hidden-terminal effects and multimedia support for wireless LAN, Computer Communications 23 (2000) 998-1013.

[7] M. Veeraraghavan, N. Cocker, T. Moors, Support of voice services in IEEE 802.11 wireless LANs, Proceedings of INFOCOM (2001) 488-497.

[8] J.L. Sobrino, A.S. Krishnakumar, Real-time traffic over the IEEE 802.11 medium access control layer, Bell Labs Technical Journal (1996) $172-187$.

[9] C. Romans, J. Tourrilhes, A. Medium, Access Protocol for Wireless LANs Which Supports Isochronous and Asynchronous Traffic, Technical Report HPL-98-35, February, Hewlett-Packard Company, Bristol, 1998.

[10] J. Deng, R.S. Chang, A priority scheme for IEEE 802.11 DCF access method, IEICE Transactions Communications E82-B (1) (1999) 96-102.

[11] S.-T. Sheu, T.-F. Sheu, A bandwidth allocation/sharing/extension protocol for multimedia over IEEE 802.11 ad hoc wireless LANs, IEEE Journal on Selected Areas in Communications 19 (10) (2001) $2065-2080$

[12] H.-H. Liu, J.-L.C. Wu, Packet telephony support for the IEEE 802.11 wireless LAN, IEEE Communications Letters 4 (9) (2000) 286-288.

[13] A. Zahedi, K. Pahlavan, Capacity of a wireless LAN with voice and data services, IEEE Transactions on Communications 48 (7) (2000) $1160-1170$.

[14] IEEE Std 802.11b-1999, Part 11: wireless LAN medium access control (MAC) and physical layer (PHY) specifications: higher-speed physical layer extension in the $2.4 \mathrm{GHz}$ band, IEEE, 1999, September.

[15] P.T. Brady, A modified for generating ON-OFF speech patterns in two-way conversation, Bell System Technical Journal 48 (7) (1969) $2445-2472$.

[16] K. Sriram, T.G. Lyons, Y.T. Wang, Anomalies due to delay and loss in AAL2 packet voice systems: performance models and methods of mitigation, IEEE Journal on Selected Areas in Communications 17 (1) (1999) 4-17.
[17] M. Fine, F.A. Tobagi, Packet voice on a local area network with round robin service, IEEE Transactions on Communications COM-34 (9) (1986) 906-915.

[18] R. O’Onvural, Asynchronous Transfer Mode Networks: Performance Issues, Artech House, Boston, 1994, pp. 37-92.

[19] N.S. Jayant, S.W. Christensen, Effects of packet losses in waveform coded speech and improvements due to an odd-even sampleinterpolation procedure, IEEE Transactions on Communications COM-29 (2) (1981) 101-109.

[20] E. Friedman, C. Ziegler, Packet voice communications over PC-based local area networks, IEEE Journal on Selected Areas in Communications 7 (2) (1989) 211-218.

Eustathia Ziouva was born in Athens, Greece in 1970. She received the Electrical Engineering Diploma in 1993 from the University of Patras, Electrical Engineering Department. In September 1993, she joined the Laboratory of Electromagnetics, where she worked in R\&D projects of the Greek Government and the European Union for 3 years as a research member. In September 1999, she started studies for $\mathrm{PhD}$ in the fields of wireless networks. Her research interests are in the areas of communication networks performance analysis. Ms Ziouva is a member of the Technical Chamber of Greece.

Theodore Antonakopoulos was born in Patras, Greece in 1962. He received the Electrical Engineering Diploma degree in 1985, and his $\mathrm{PhD}$ degree in 1989 from the Department of Electrical Engineering at the University of Patras, Patras, Greece. In September 1985, he joined the Laboratory of Electromagnetics at the University of Patras participating in various $R \& D$ projects for the Greek Government and the European Union, initially as a research staff member and subsequently as the senior researcher of the Communications Group. Since 1991, he has been on the Faculty of the Electrical Engineering Department at the University of Patras, where he is currently an Associate Professor. His research interests are in the areas of data communication networks with emphasis on performance analysis, efficient hardware implementation and rapid prototyping. He has more than 70 publications in the above areas and is actively participating in several R\&D projects of the European industry. Dr Antonakopoulos is a Senior member of the IEEE, serves in the Program Committee of various international Conferences and Workshops and is a member of the Technical Chamber of Greece. 